\title{
Cutaneous mucormycosis of the lower extremity leading amputation in two diabetic patients
}

\author{
Kathleen M. Coerdt ${ }^{1}$, Elizabeth G. Zolper², Amy G. Starr ${ }^{3}$, Kenneth L. Fan², \\ Christopher E. Attinger ${ }^{2}$, Karen K. Evans ${ }^{2}$ \\ ${ }^{1}$ Georgetown University School of Medicine, Washington, D.C.; Departments of ${ }^{2}$ Plastic and Reconstructive Surgery and ${ }^{3}$ Pathology, MedStar \\ Georgetown University Hospital, Washington, D.C., USA
}

\begin{abstract}
Mucormycosis is an invasive, rapidly progressive, life-threatening fungal infection, with a propensity for diabetic, immunosuppressed, and trauma patients. The classic rhinocerebral variation is most common in diabetic patients. While the cutaneous form is usually caused by direct inoculation in immunocompetent patients. Cutaneous mucormycosis manifests in soft tissue and risks involvement of underlying structures. Tibial osteomyelitis can also occur secondary to cutaneous mucormycosis but is rare. Limb salvage is typically successful after lower extremity cutaneous mucormycosis even when the bone is involved. Herein, we report two cases of lower extremity cutaneous mucormycosis in diabetic patients that presented as acute worsening of chronic pretibial ulcers. Despite aggressive antifungal therapy and surgical debridement, both ultimately required amputation. Such aggressive presentation has not been reported in the absence of major penetrating trauma, recent surgery, or burns.
\end{abstract}

Keywords Mucormycosis / Leg ulcer / Amputation / Diabetes mellitus / Wound infection

\author{
Correspondence: Karen K. Evans \\ Department of Plastic and \\ Reconstructive Surgery, MedStar \\ Georgetown University Hospital, 3800 \\ Reservoir Road, One Bles, Washington, \\ D.C. 20007 , USA \\ Tel: +1-202-444-3059 \\ Fax: +1-202-444-0300 \\ E-mail: Karen.K.Evans@medstar.net
}

Received: March 28, $2020 \bullet$ Revised: August 8, $2020 \bullet$ Accepted: August 12, 2020

pISSN: 2234-6163 • elSSN: 2234-6171 • https://doi.org/10.5999/aps.2020.00549 • Arch Plast Surg 2021;48:231-236

\section{INTRODUCTION}

Mucormycosis is a rare, life-threatening fungal infection that typically progresses rapidly [1-3]. It is caused by filamentous fungi belonging to the Mucorales order; the pathogens most commonly responsible for human disease are Rhizopus species (spp.) [1-4]. Other human pathogens include Mucor, Cunninghamella, Absidia, Lichtheimia, and Apophysomyces spp. with greater than 20 human pathogens identified $[1,4]$. Prior literature estimates the annual incidence of mucormycosis is less than two cases per million individuals [3]. There is evidence to support an increasing incidence of mucormycosis as the population at-risk has grown significantly $[3,4]$. However, it remains difficult to determine the true incidence of mucormycosis as many suspected cases lack definitive mycologic or histologic evidence $[1,3,4]$.

Patients at-risk of mucormycosis are typically immunocompromised especially those with uncontrolled diabetes, hematologic malignancies, and undergoing solid organ or hematopoietic stem cell transplantation [2,3]. Immunocompetent patients experiencing major penetrating trauma and burns are also predisposed to mucormycosis [2,3]. Most infections are community-acquired and occur via inhalation of fungal spores or direct inoculation via violations in the cutaneous barrier $[2,3]$. Reports of hospital-associated mucormycosis are becoming more common; contaminated catheters, dressings, and oral medications have been implicated [4]. Mucormycosis is divided into six forms based on the anatomic site involved: rhinocerebral, 
pulmonary, cutaneous, gastrointestinal, disseminated, and uncommon presentations [2]. All forms are characterized by angioinvasion, which manifests clinically as vessel thrombosis and tissue necrosis, and carry a high risk of mortality [2]. In the diabetic population, most commonly affected by rhinocerebral mucormycosis, the mortality rate is reported to be $44 \%$ [3].

Cutaneous mucormycosis, the third most common form, often affects immunocompetent patients [4]. Only $10 \%$ to $15 \%$ of cases are reported to involve diabetic hosts [4]. The most common risk factor, implicated in up to $88 \%$ of cases, is penetrating trauma with soil being the source of inoculation $[2,4]$. Burns, surgery, persistent maceration, and subcutaneous or intramuscular injections are also risk factors $[2,4]$. Cutaneous infection can remain localized, invade deeper structures (e.g., muscle, tendons, and bones), or disseminate to other organs [4]. When deep extension occurs, it usually presents as necrotic eschar with surrounding erythema and necrotizing infection [4]. However, cutaneous mucormycosis may also present indolently with skin findings limited to a small erythematous macule [3]. Urgent, aggressive surgical debridement is the cornerstone of treatment for all presentations of cutaneous mucormycosis [2]. Prompt diagnosis, control of predisposing factors, and appropriate antifungal treatment are also essential [2]. We report two cases of lower extremity cutaneous mucormycosis with deep extension in diabetic patients. These are the first reported cases

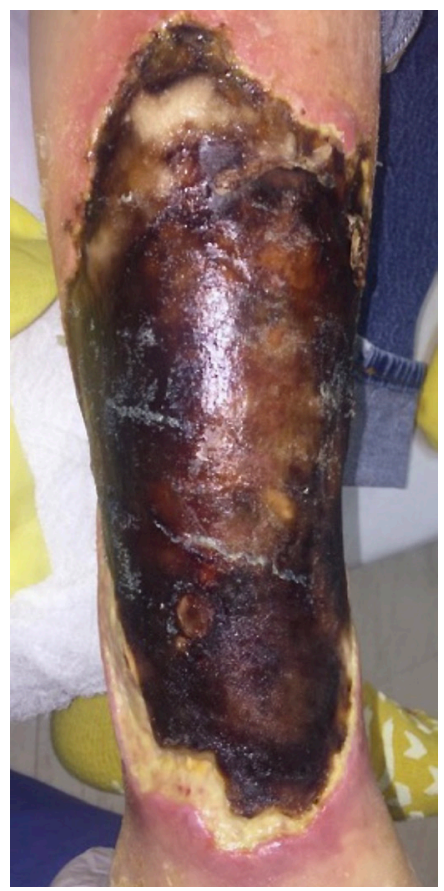

Fig. 1. Classic findings. Case 1 presented with a $22 \times 14 \mathrm{~cm}$ anterior right lower leg wound with significant eschar without signs of systemic illness. The wound began as a small skin tear secondary to a fall 2 months prior. of cutaneous mucormycosis resulting in amputation in the absence of major penetrating trauma, recent surgery, or burns. Institutional Review Board approval was obtained (IRB No. 2018173) for review of patient records. Both patients provided informed consent for the publication of their clinical case and the accompanying preoperative, intraoperative, and postoperative photographs.

\section{CASES}

\section{Case 1}

A 60-year-old male patient presented with eschar of the anterior right lower leg measuring $22 \times 14 \mathrm{~cm}$ (Fig. 1). There were no signs of systemic illness. He had multiple comorbidities (Charlson comorbidity index [CCI] of 9) [5] including diabetes mellitus, peripheral neuropathy, chronic kidney disease (CKD), and congestive heart failure. He reported incurring a small skin tear secondary to a fall 2 months prior. However, the wound had acutely worsened after being discharged from an outside hospital 4 days prior. The primary reason for admission had been confusion and generalized weakness attributed to pneumonia and worsening CKD. However, he also received intravenous vancomycin and cefepime due to concern for cellulitis.

At admission, tibia/fibula radiographs showed soft tissue irregularity consistent with superficial ulceration and no features of osteomyelitis. Intravenous doxycycline was initiated for empiric coverage. Surgical debridement on the 2nd day of admission (DOA), revealed frank purulence beneath the eschar with necrotic tissue throughout the entire anterior compartment down to tibial periosteum (Fig. 2). At this time, preliminary cul-
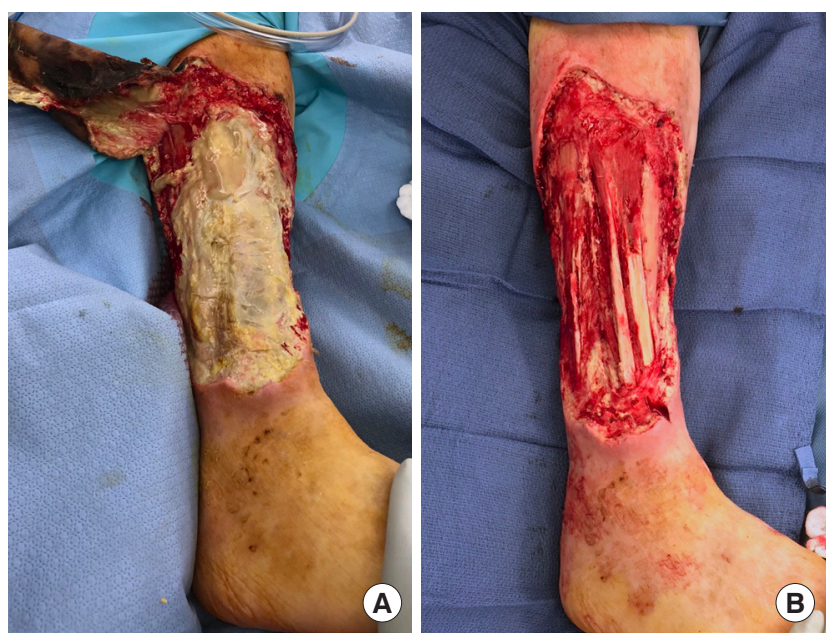

Fig. 2. Initial debridement of case 1 wound. Frank purulence beneath the eschar with necrotic tissue throughout the entire anterior compartment down to tibial periosteum (A) was appreciated requiring aggressive surgical debridement (B). 
ture results revealed light growth of fungus, yeast, and coagulase negative staphylococcus. Intravenous fluconazole was added to the patient's medication regimen until further speciation results became available. On DOA 6, final culture results identified the fungus as Rhizopus. On DOA 7, preliminary pathology found marked necrosis and invasive fungal infection with hyphae; thus, the antifungal was changed to micafungin. On DOA 8, final pathology revealed features suggestive of mucormycosis including numerous irregularly shaped hyphae, scattered necroticappearing vessels, diffuse Periodic acid-Schiff staining, and poor Grocott-Gomori's methenamine silver staining [6].

However, micafungin was maintained in effort to spare the patient's remaining renal function as there was no fungal growth on repeat cultures. After consultation with dermatology and nephrology, it was ultimately decided that the benefits outweighed the risks. Thus, the antifungal regimen was changed to liposomal amphotericin B (LAmB) on DOA 10. Despite continued aggressive debridement, $\mathrm{LAmB}$, and the addition of isavuconazole, repeat pathology on DOA 21 and 27 both showed invasive mucormycosis throughout the soft tissue.

Due to continued positive margins after serial debridement, worsening appearance, and after consultation with the mycology division at the National Institutes of Health, a knee disarticulation was performed on DOA 39. Pathology was negative for fungal elements indicating clean margins. There was no evidence of osteomyelitis on the patella. After stabilization of medi-

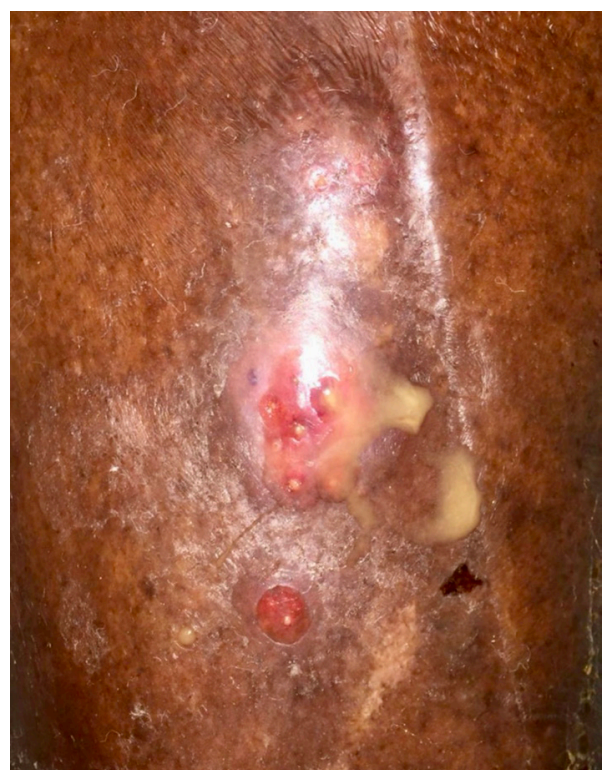

Fig. 3. Atypical findings. Case 2 presented with a mid-tibial ulcer in the absence of known trauma which is atypical for cutaneous mucormycosis. The ulcer had been present for about 4 months and began blistering and draining purulent fluid a week prior to presentation. cal complications, he was discharged to acute rehabilitation 12 weeks after admission with 4 weeks of oral posaconazole remaining to complete a 10-week course.

\section{Case 2}

A 77-year-old male patient presented with a left mid-tibial ulcer in the absence of known trauma (Fig. 3). He had been living in a rehabilitation facility since undergoing a right below knee amputation 3 years prior. He was legally blind and had multiple comorbidities (CCI of 7) including diabetes mellitus, end-stage renal disease on dialysis, atrial fibrillation on anticoagulation, peripheral vascular disease, and hypothyroidism. The ulcer had been present for 4 months with new onset of blistering and purulent drainage a week prior to presentation. Outside imaging from 25 days prior to presentation was revealed circumferential periostitis with cortical erosions and abnormal bone marrow signal involving the tibia and head of fibula. Thus, he had been receiving vancomycin during dialysis due to high suspicion of osteomyelitis.

At admission, the patient had an elevated erythrocyte sedimentation rate and C-reactive protein above $120 \mathrm{mg} / \mathrm{L}$. Initial debridement demonstrated widespread purulence around the tibia and erosion of the bony cortex. Over 20 cultures were collected at this time which were all initially negative. Repeat debridement on DOA 8, revealed a clean wound (Fig. 4). However, a single culture began to grow mold on DOA 9; thus, vori-

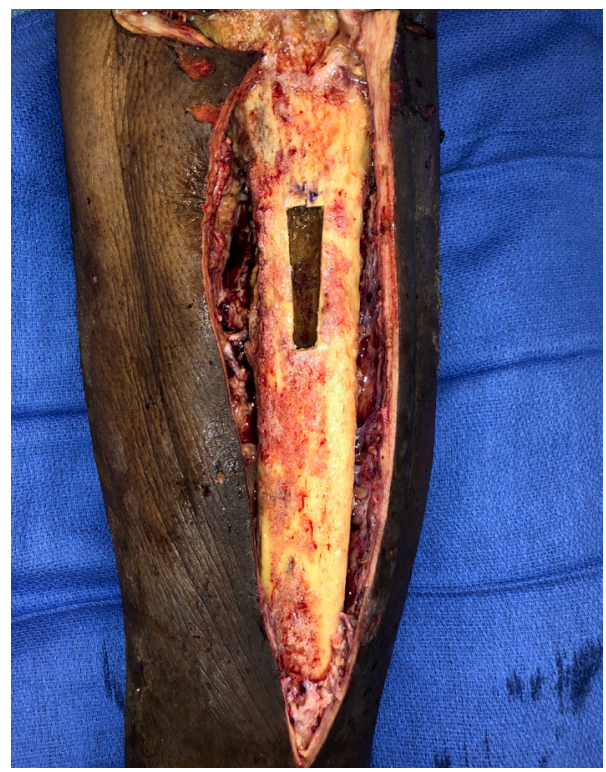

Fig. 4. Initial debridement of case 2 wound. There were widespread pitting and infection of the tibia that appeared non-salvageable. At this time, the knee joint appeared clear and preliminary plans for a stage below knee amputation were made pending cultures and pathology. 


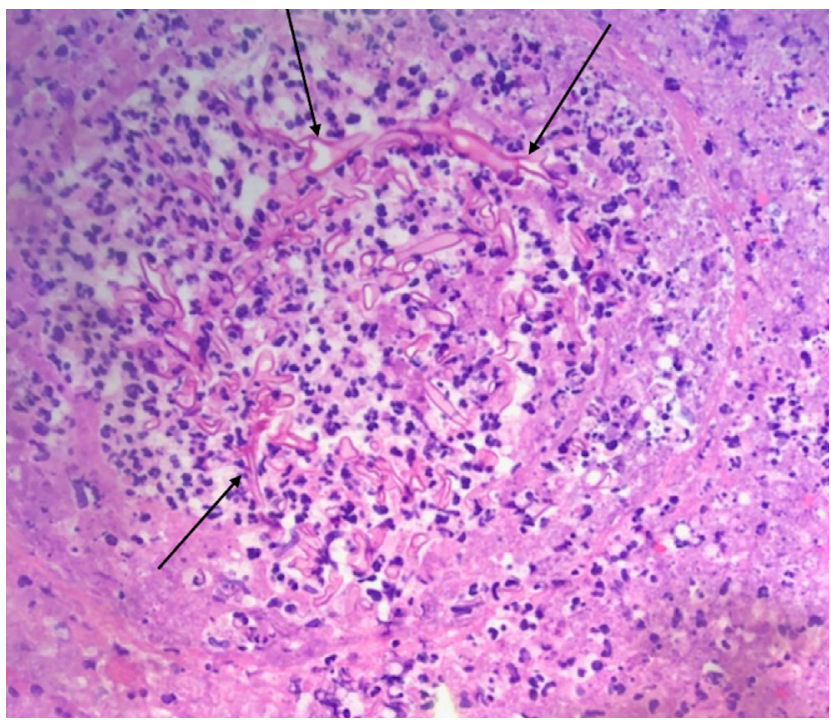

Fig. 5. Left foot biopsy demonstrating Mucorales. Nodular focus of fungal organisms with wide, ribbon-like hyphae with right angle branching (black arrows) and surrounding acute inflammatory infiltrate $\left(H \& E_{1} \times 40\right)$.

conazole was started empirically. By DOA 11, both Mucorales and Aspergillus had been identified in various cultures and the antifungal was switched to posaconazole for better Mucorales coverage.

Given the diagnosis of Mucorales osteomyelitis, the decision was made to proceed with amputation. However, there was extension of purulence in the proximal prepatellar region and persistence of fungal elements on intraoperative pathology (Fig. 5). Thus, an above knee amputation with excision of the distal quadriceps muscle was performed on DOA 18. Pathology confirmed clean margins. On DOA 29, final speciation identified Lichtheimia corymbifera with reasonable sensitivity to voriconazole (minimum inhibitory concentration $[\mathrm{MIC}], 4 \mu \mathrm{g} / \mathrm{mL}$ ) but resistance to posaconazole ( $\mathrm{MIC} \geq 16 \mu \mathrm{g} / \mathrm{mL}$ ). Thus, the patient was initiated on oral voriconazole and discharged with a 3-month course.

\section{DISCUSSION}

Cutaneous mucormycosis remains uncommon with less than 200 reported cases identified in a recent meta-analysis [7]. Of those, deep extension occurred in only 37 cases. Limb loss in the setting of cutaneous mucormycosis is even less common. To date, there have been three reports of progression to amputation; all of these cases involved significant disruptions in the cutaneous barrier in the form of trauma, surgery, or burn wounds [8-10]. However, our cases are unique because deep extension of mucormycosis and limb loss was preceded by seemingly mundane superficial wounds. There was no history of recent surgery or trauma which would have facilitated inoculation of deeper structures. Challenges in diagnosis and the highly comorbid nature of these patients both contributed to the poor outcome. Despite this, both patients healed well without further complications and received prosthetics at 4 months after their amputation.

In our first case, mucormycosis presented with classic findings of necrotic eschar and erythema. Light growth of Rhizopus was identified on day 6 of admission. However, the likelihood of Rhizopus as a contaminant and the risk of nephrotoxicity delayed initiation of amphotericin B until day 10 of admission. Progressive necrosis and inability to achieve clean margins necessitated amputation. This is similar to the case reported by Stanistreet and Bell [8] in 2017, where widespread necrosis of the lower extremity developed after infection of a burn wound. In that case the diagnosis of mucormycosis was delayed for over 40 days, by which time the patient had globally deteriorated and subsequently expired despite the initiation of antifungal therapy.

Our second case had an atypical presentation with skin findings limited to a small ulcer with new purulent drainage. However, deep extension to the tibia had already occurred. Such a discrepancy in severity between cutaneous findings and overall extent of mucormycosis has been reported to occur in immunosuppressed patients [3]. In this case, diagnosis of mucormycosis was made on day 11 of admission spurring a change in antifungal regimen from voriconazole to posaconazole as Mucorales is reported to be resistant to voriconazole [11]. Unexpectedly, final sensitivities revealed complete resistance to posaconazole but reasonable sensitivity to voriconazole. In a prior case of tibial osteomyelitis resulting from contiguous spread of mucormycosis from a pretibial ulcer, limb salvage was successful after surgical debridement and treatment with amphotericin B [12]. The delay in effective antifungal coverage likely contributed to residual mucormycosis which necessitated more proximal amputation.

Delays in diagnosis and adequate treatment were significant factors in both cases. The relatively insidious nature of both presentations also resulted in a delay in seeking care. In fact, one of the infected ulcers was noted at a routine follow up appointment for a contralateral below knee amputation. Diagnosis traditionally relies on both cultures and histopathology $[6,11]$. When a patient comes in with a chronic purulent wound, fungal cultures should be sent immediately. Fungus on wound cultures may be an environmental contaminant; thus, correlation with histopathology is essential to confirm fungus as a pathogen $[6,11]$. Fungal cultures can also be falsely reassuring as they are only positive in $50 \%$ cases confirmed by histopathology [11]. Given the need to confirm positive fungal cultures with histopa- 
thology, it is imperative that tissue samples for culture and histopathology be collected from the same area [6]. This avoids inadvertently sampling a second area without fungal elements, which if sent to histopathology risks incorrectly concluding a positive fungal culture is due to contamination.

Early diagnosis and treatment are vital to decrease morbidity and mortality associated with mucormycosis; research has focused on new diagnostic methods [11]. Molecular based techniques, such as polymerase chain reaction, can be utilized for detection and identification of Mucorales [1,11]. Most mucormycosis pathogens are identified based on morphology; however, molecular studies are necessary for accurate identification of some species [1]. Morphologically indistinguishable Lichtheimia isolates previously thought to all be L corymbifera were determined to be two unique species based on divergent internal transcribed spacer sequences [1]. Use of internal transcribed spacer sequencing to confirm morphologic identification of $L$. corymbifera in the clinical setting has been described in a recent case report [13].

The clinical utility of such molecular assays may be limited as prior literature reported no significant difference in antifungal sensitivities of Lichtheimia spp. requiring molecular studies for speciation [1]. However, the unusual antifungal sensitivities of L. corymbifera in our second case demonstrate the value of molecular studies as faster speciation would have significantly altered clinical management. A recently published study on species-specific antifungal susceptibility profiles found the MIC of posaconazole for eight $L$. corymbifera isolates was 0.1 ; thus, we believe this pattern of posaconazole resistance has not been previously reported [14]. In the first patient's case, sensitivity assays were not performed as LAmB, which is the drug of choice for mucormycosis, was utilized. However, susceptibility to amphotericin B does vary between species [14]. In a 2014 case report, cutaneous mucormycosis caused by an isolate of Apophysomyces with a high MIC to amphotericin B led to the patient's death due to nonresponse to amphotericin B and fluconazole [15]. Sensitivities may also have been of value in selecting a second antifungal agent when disease progression continued despite appropriate management.

Given the unprecedented aspects of these cases, mucormycosis warrants increased clinical suspicion, especially in diabetic wound patients with atypical presentations. In such patients, it is obligatory to send specimens for acid-fast bacilli and fungal cultures. It remains unclear whether the poor outcomes we report represent a "perfect storm" of host factors and diagnostic delays or if healthcare-associated Mucorales with increased virulence may have contributed as both patients had recent, prolonged exposure to healthcare facilities. Despite remaining an uncommon disease entity, increased awareness of the spectrum of potential presentations as well as the evolving nature of mucormycosis diagnosis and management is critical to minimize morbidity and mortality.

\section{NOTES}

\section{Conflict of interest}

No potential conflict of interest relevant to this article was reported.

\section{Ethical approval}

The study was approved by the Institutional Review Board of MedStar Health Research Institute (IRB No. 2018-173) and performed in accordance with the principles of the Declaration of Helsinki. Written informed consent was obtained.

\section{Patient consent}

The patients provided written informed consent for the publication and the use of their images.

\section{Author contribution}

Conceptualization: KM Coerdt, EG Zolper, AG Starr, KL Fan, CE Attinger, KK Evans. Data curation: KM Coerdt, EG Zolper, AG Starr, KL Fan, CE Attinger, KK Evans. Visualization: A Starr, KK Evans. Writing - original draft: KM Coerdt, EG Zolper. Writing - review \& editing: KM Coerdt, EG Zolper, AG Starr, KL Fan, CE Attinger, KK Evans.

\section{ORCID}

Kathleen M. Coerdt https://orcid.org/0000-0003-0631-9779 Elizabeth G. Zolper https://orcid.org/0000-0001-6271-6918 Amy G. Starr https://orcid.org/0000-0001-9894-4058 Kenneth L. Fan https://orcid.org/0000-0001-5951-5576 Christopher E. Attinger

https://orcid.org/0000-0001-7114-6275

Karen K. Evans https://orcid.org/0000-0003-1056-2114

\section{REFERENCES}

1. Garcia-Hermoso D, Hoinard D, Gantier JC, et al. Molecular and phenotypic evaluation of Lichtheimia corymbifera (formerly Absidia corymbifera) complex isolates associated with human mucormycosis: rehabilitation of L. ramosa. J Clin Microbiol 2009;47:3862-70.

2. Spellberg B, Edwards J Jr, Ibrahim A. Novel perspectives on mucormycosis: pathophysiology, presentation, and management. Clin Microbiol Rev 2005; 18:556-69. 
3. Petrikkos G, Skiada A, Lortholary O, et al. Epidemiology and clinical manifestations of mucormycosis. Clin Infect Dis 2012;54 Suppl 1:S23-34.

4. Prakash H, Chakrabarti A. Global epidemiology of mucormycosis. J Fungi (Basel) 2019;5:26.

5. Charlson M, Szatrowski TP, Peterson J, et al. Validation of a combined comorbidity index. J Clin Epidemiol 1994;47: 1245-51.

6. Guarner J, Brandt ME. Histopathologic diagnosis of fungal infections in the 21st century. Clin Microbiol Rev 2011;24: 247-80.

7. Jeong W, Keighley C, Wolfe R, et al. The epidemiology and clinical manifestations of mucormycosis: a systematic review and meta-analysis of case reports. Clin Microbiol Infect 2019;25:26-34.

8. Stanistreet B, Bell D. Burn wound mucormycosis: a case study on poor wound healing.J Burn Care Res 2017;38:e582-4.

9. Holtom PD, Obuch AB, Ahlmann ER, et al. Mucormycosis of the tibia: a case report and review of the literature. Clin
Orthop Relat Res 2000;(381):222-8.

10. Pierce PF, Wood MB, Roberts GD, et al. Saksenaea vasiformis osteomyelitis. J Clin Microbiol 1987;25:933-5.

11. Skiada A, Lass-Floerl C, Klimko N, et al. Challenges in the diagnosis and treatment of mucormycosis. Med Mycol 2018;56(suppl_1):93-101.

12. Maliwan N, Reyes CV, Rippon JW. Osteomyelitis secondary to cutaneous mucormycosis: report of a case and a review of the literature. Am J Dermatopathol 1984;6:479-81.

13. Tyll T, Lyskova P, Hubka V, et al. Early diagnosis of cutaneous mucormycosis due to Lichtheimia corymbifera after a traffic accident. Mycopathologia 2016;181:119-24.

14. Wagner L, de Hoog S, Alastruey-Izquierdo A, et al. A revised species concept for opportunistic Mucor species reveals species-specific antifungal susceptibility profiles. Antimicrob Agents Chemother 2019;63:e00653-19.

15. Bonifaz A, Stchigel AM, Guarro J, et al. Primary cutaneous mucormycosis produced by the new species Apophysomyces mexicanus. J Clin Microbiol 2014;52:4428-31. 